\title{
Carbon nanotube modified electrodes for enhanced brightness in organic light emitting devices
}

\author{
A.R. Inigo, J.M. Underwood and S.R.P. Silva* \\ Nanoelectronics Center, Advanced Technology Institute, \\ University of Surrey, \\ Guildford, GU2 7XH, Surrey, UK. ${ }^{1}$
}

Keywords: OLED, CNT, MWCNT, brightness, hole injection

\begin{abstract}
Acid oxidized multiwall carbon nanotubes (COOH-MWCNT) have been introduced as a hole injection buffer layer in an organic light emitting diodes (OLED). We show that the OLED with COOH-MWCNT as a buffer layer provides higher brightness with lower operating voltages. The addition of a $\mathrm{COOH}-\mathrm{MWCNT}$ buffer layer has enabled a further increase in the brightness of our OLED devices operating in excess of $20,000 \mathrm{~cd} / \mathrm{m}^{2}$ due to enhanced hole injection by several orders of magnitude. The increase in current injection and brightness does not alter the optical emission spectrum at different operating voltages in these devices. A model is proposed to explain this increase in current injection and brightness based on the modified electron energy band alignment.
\end{abstract}

*Corresponding Author Email: s.silva@surrey.ac.uk 


\section{Introduction}

High quality OLEDs has been realised by designing multilayer structures with balanced electron and hole injection, and efficient transport into the emitting layer using specific transport materials [1]. N,N'-diphenyl-N,N -bis(3 -methyl-phenyl)- 1,1'biphenyl-4,4 diamine (TPD) is one among the many favoured hole transport materials due to its ability to act as a hole injector because of its favourable energy level positions at the anode $[2,3]$. Likewise, Tris(8-hydroxyquinolinato)aluminium (Alq3) is one of the few efficient electron transport organic materials due to its relatively high electron mobility and its favourable energy level positions at the cathode [4]. For better hole and electron injection, further research is focussed on incorporating single wall (SW) and multiwall (MW) carbon nanotubes (CNT) [5-8]. CNT have been introduced into the OLED structures in the form of composites [9] or as a single layer from a solution functionalised CNT spin coated on to the top of an anode [10] or spin coated prior to evaporation of the cathodes [11].

Indium tin oxide (ITO) which has a work function of $\sim 4.7 \mathrm{eV}$ is widely used as an anode due to its high optical transmittance and high sheet conductance. A threshold barrier height of $\sim 0.3-0.4 \mathrm{eV}$ or lower with respect to the adjacent hole transporting layer (HTL) is required for the OLED to operate with space charge limited current (SCLC) and barriers higher than $0.3 \mathrm{eV}$ lead to injection limited current [12]. However, ITO's work function of 4.7 $\mathrm{eV}$ produces a barrier height higher than $0.3 \mathrm{eV}$ for most hole transporting materials which have a highest occupied molecular orbital around 5.0 to $5.5 \mathrm{eV}$ [12,13]. To overcome this barrier, buffer layers such as copper phthalocyanine [6], poly $(3,4-$ ethylenedioythiophe):poly(styrenesulphonate) (PEDOT:PSS) and PEDOT:PSS:CNT composites have been introduced between the ITO and the HTL $[9,13]$. Whilst the performance improvements from these HTLs are moderate, the conventional HTLs have shown to introduce other complexities into the device stack. The widely used PEDOT:PSS is 
acidic in nature, known to affect the underlying ITO layer which is a major disadvantage. Therefore, cheaper, solution processable HTL candidates such as CNTs show promise in this regard.

While introduction of various HTLs have been demonstrated to give improved performance, introduction of $\mathrm{CNT}$ as a barrier reduction buffer layer at the anode or at the cathode is still untested and at the demonstration level. For example, the reference ITO/HTL/Alq3/Al have demonstrated operation up to 15 to $20 \mathrm{~V}$ with the highest luminance of $4000 \mathrm{~cd} / \mathrm{m}^{2}$ at the driving voltage of $\sim 20 \mathrm{~V}$, and, with the introduction of PEDOT:PSS:MWCNT as an anode buffer layer, the luminance increased up to $6500 \mathrm{~cd} / \mathrm{m}^{2}$ at the driving voltage of $\sim 16 \mathrm{~V}$ with the operating voltage of 10 to $16 \mathrm{~V}$ [9]. Furthermore, CNT has been used as a cathode buffer layer to achieve the maximum luminance of the reference device from $\sim 1000 \mathrm{~cd} / \mathrm{m}^{2}$ to $\sim 5000$ $\mathrm{cd} / \mathrm{m}^{2}$ at the driving voltage of $15 \mathrm{~V}$ with the operating voltage of 8 to $15 \mathrm{~V}$ [11]. Here, we present a green light emitting device with $\mathrm{COOH}-\mathrm{MWCNT}$ as the buffer layer in between ITO and the HTL.

In this work, OLEDs were fabricated with TPD as a hole transport layer and Alq3 as an emitting layer. The reference ITO anode OLED has the device architecture of ITO/TPD(35nm)/Alq3(35nm)/LiF/Al. OLED with ITO/COOH-MWCNT as an electrode has the architecture of ITO/COOH-MWCNT/TPD(35nm)/Alq3(35nm)/LiF/Al. Hole only single layer devices were also prepared with ITO/TPD(70nm)/Al and ITO/COOHMWCNT/TPD(70nm)/Al to compare the current injection characteristics with that of corresponding OLEDs.

\section{Experimental}

\section{1 Acid functionalization of MWCNTs}


250mg MWCNT (Sigma Aldrich) were added to $15 \mathrm{ml}$ of a 3:1 mixture of concentrated Sulphuric:Nitric acid (Fisher Scientific AR grade) in a round bottom flask and mixed for 10 minutes in an ultrasonic bath. After sonication the mixture was refluxed over an oil bath at $130^{\circ} \mathrm{C}$ for 1 hour. After allowing the mixture to cool it was carefully diluted to $80 \mathrm{ml}$ using MilliQ deionised water and transferred into two $50 \mathrm{ml}$ centrifuge tubes and centrifuged at 8500rpm (approx 10000xg) for $20 \mathrm{~min}$. The supernatant (brown liquid) was removed leaving a black precipitate which was then diluted with MilliQ DI water and the precipitate suspended using a vortex mixer. This process was repeated twice to remove most of the concentrated acid used in the reaction leaving a black liquid with no obvious phases. Centrifuging was continued for 20 minutes at a time and the black liquid decanted into clean centrifuge tubes until no obvious precipitate remained.

This solution is filtered over $0.1 \mu \mathrm{m} 47 \mathrm{~mm}$ polycarbonate membrane filter whilst washing with DI water until approx $\mathrm{pH} 7$ is reached and finally washed with absolute Ethanol (Fisher AR grade). This final product of $\mathrm{COOH}-\mathrm{MWCNT}$ is dried in vacuum dessicator and dry weight obtained. The dry COOH-MWCNT was re-dispersed into 50\% Ethanol/DI water at concentration of $10 \mathrm{mg} / \mathrm{ml}$ over a period of 4 days on an orbital mixer. Fig. 1 shows a scanning electron microscopy image of a typical MWCNT film, which illustrates the discontinuous nature of the layer.

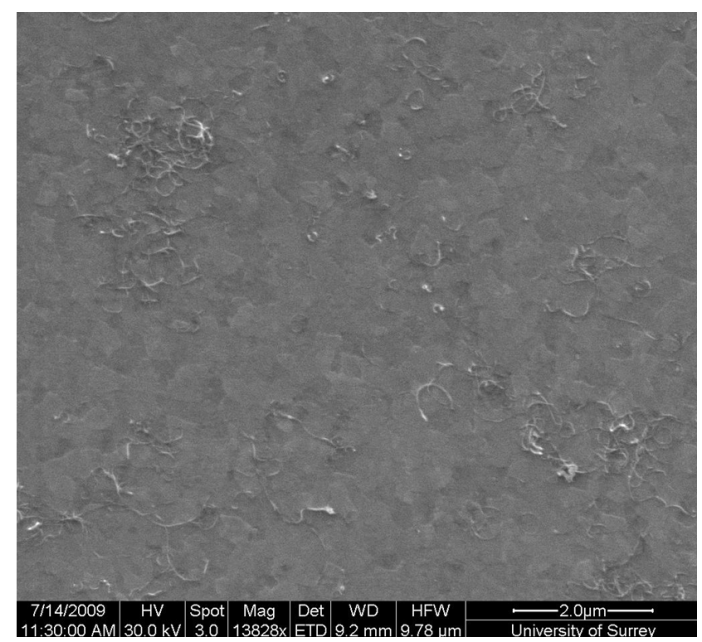


Figure 1. Scanning electron micrographs of as functionalised MWCNTs spin cast at 1000rpm for a minute from aqueous solution on ITO coated glass substrates.

\subsection{Device fabrications}

Prior to spin coating COOH-MWCNT, ITO substrates were cleaned in an ultrasonic bath with acetone, isopropanol and methanol consecutively for about 5 minutes followed by oxygen plasma cleaning. After spin-coating COOH-MWCNT, the films were dried at $100^{\circ} \mathrm{C}$ to remove water and ethanol. These COOH-MWCNT coated substrates were loaded into a vacuum chamber. TPD $(35 \mathrm{~nm}), \mathrm{Alq} 3(35 \mathrm{~nm}), \mathrm{LiF}$ and $\mathrm{Al}$ were evaporated under the evaporation pressure of $1 \times 10^{-5}$ torr. Active device area was defined as $5 \mathrm{~mm}^{2}$ using a shadow mask just before the $\mathrm{Al}$ evaporation, resulting in three devices on a single substrate. To compare the hole injection in these devices, single layer devices of TPD (70 nm) were also prepared with Al cathode. The schematic diagram with the proposed energy levels is shown in Fig. 2 (a-d). Fabricated devices were briefly exposed during the transfer from thermal evaporator to the vacuum cryostat. All the devices were tested under continuous driving voltage with $0.1 \mathrm{~V}$ step in a vacuum cryostat at room temperature with calibrated Silicon photo-diode. The experiment was repeatable consistently, with acceptable performance deviations suggested to be due to the random distribution of MWCNTs on the ITO electrode.

(a)

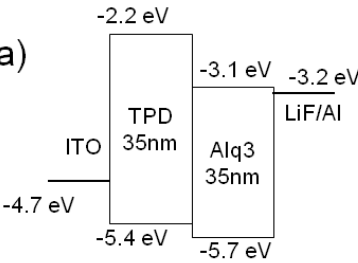

(c)

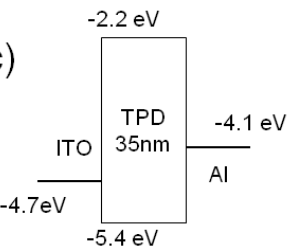

(b)

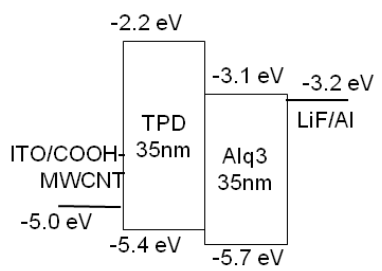

(d)

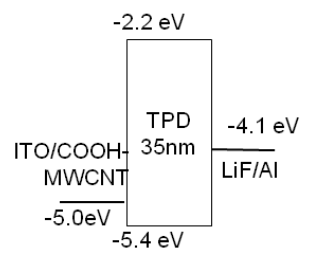


Figure 2. Schematic diagram with energy levels of (a) ITO reference electrode OLED

(b) ITO/COOH-MWCNT electrode OLED (c) ITO reference electrode hole only device and (d) ITO/COOH-MWCNT electrode hole only device.

\section{Results and Discussion}

Charge carrier injection efficiency in the reference ITO and COOH-MWCNT modified ITO devices can be determined from the ratio of the measured current density in these devices to a space charge limited current density. The theory of SCLC was proposed originally to explain the charge transport characteristics in insulating solids $[14,15]$. As organic semiconducting materials have shallow and deep traps, this SCLC has been extended to describe them [16]. At sufficiently high injection, all the traps would be filled and the conduction will become trap-free SCL. The SCLC in organic semiconductors under the trap free conditions is given by Mott-Gurney law as;

$$
J_{T F S C L C}=\frac{9}{8} \varepsilon \varepsilon_{0} \mu \frac{E^{2}}{d}
$$

where $\mathbf{J}_{\text {TFSCLC }}$ is the trap free space charge limited current, $\varepsilon$ is the relative dielectric constant, $\varepsilon_{0}$ is the permittivity of the vacuum and $\mu$ is the mobility. Hence, the injection efficiency $(\eta)$ can be written as,

$$
\eta=\frac{J}{J_{T F S C L C}}
$$

where $\mathbf{J}$ is the measured current density. In general, injection efficiencies are measured in two steps on single layer devices and mostly on hole transport materials having thicknesses of the order of $\mu \mathrm{m}$ [17-21]. In such cases, the hole mobility measured by time of flight technique [18] or by dark injection currents [19] is used to calculate the SCL current. Then the injected current $\left(\mathrm{J}_{\mathrm{inj}}\right)$ is measured directly from $\mathrm{J}-\mathrm{V}$ measurements. Then injection efficiency is calculated from the ratio of $\mathrm{J}_{\mathrm{inj}} / \mathrm{J}_{\mathrm{SCL}}$. 
In the present study we have used a working OLED device configuration which has the thickness around $70 \mathrm{~nm}$. In TPD/Alq3 OLED, injection occurs from both electrodes; anode and cathode. While the electron injection is the same in both devices, the hole injection varies due to the modification of electrodes at the anode. So, the variation in injection efficiencies can be assumed to be due to the presence of COOH-MWCNT modified ITO. To avoid any ambiguity of these double carrier injection devices, we have fabricated hole only devices of single layer TPD (70nm) with ITO and ITO/COOH-MWCNT anodes and Al as cathode. TPD hole mobility, $\mu_{\text {hole }}=10^{-3} \mathrm{~cm}^{2} / \mathrm{Vs}$ is used to determine the $\mathrm{J}_{\mathrm{SCL}}$ from equation (2) $[21]$.
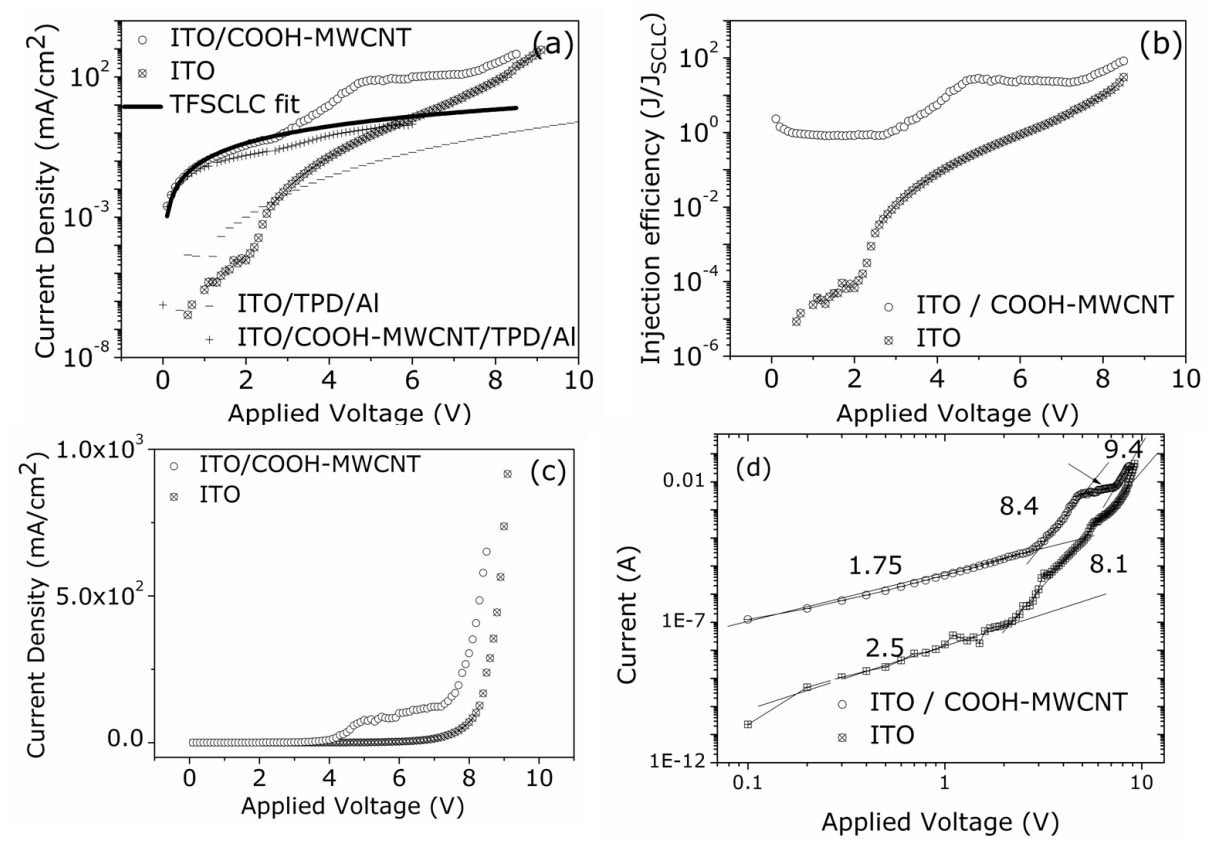

Figure 3. (a) Current density vs voltage measurements of reference ITO, ITO/COOHMWCNT electrode OLEDs and hole only devices in log - linear scale. The solid line represents SCLC. (b) Injection efficiency versus applied electric field for the reference ITO and ITO/COOH-MWCNT devices. (c) Current density vs voltage measurements of reference ITO and ITO/COOH-MWCNT electrode OLED in linear scale. (d) Current-voltage 
characteristics of ITO reference electrode OLED and ITO/COOH-MWCNT electrode OLED in log-log scale to represent I $\alpha V^{m}$ behaviour, with fitted $m$ values.

Fig. 3a shows the current density vs voltage ( $\mathrm{J}-\mathrm{V})$ characteristics of $\mathrm{J}_{\mathrm{SCL}}$, OLED and hole only devices with reference ITO and ITO/COOH-MWCNT electrodes. OLED devices follow the well established behaviour of OLEDs with an Alq3 emitter in which charge carrier transport is trap charge limited SCLC. In these devices current - voltage (I-V) characteristics was described by the power-law (I $\alpha \mathrm{V}^{\mathrm{m}}$, Fig 3d) dependence for different exponent $\mathrm{m}$ with variable operating regions ; before and after the on-set of EL [11]. This exponent $\mathrm{m}$ before the onset of EL determines the type of contact and after the EL onset is determines the type of bulk conductivity in Alq3 [22]. With voltages lower than the turn-on voltage, these devices follow a power law dependence of $\mathrm{m}=\sim 2.5$ and $\sim 1.7$ for the reference ITO and ITO/COOHMWCNT respectively and after turn on the reference ITO and ITO/COOH-MWCNT OLED follows a power law dependence with $\mathrm{m}=\sim 8$ [22].

While the $\mathrm{J}-\mathrm{V}$ of ITO/TPD/Al devices varies significantly from the calculated $\mathrm{J}_{\mathrm{SCL}}$, ITO/COOH-MWCNT/TPD/Al devices showed a closer match. This indicates that ITO/COOH-MWCNT electrodes enhances hole injection into TPD. Work function measurements on ITO and ITO/COOH-MWCNT by our group [23] using Kelvin probe method which confirms the ITO/COOH-MWCNT/TPD barrier height is within the required threshold barrier height for SCLC ( i.e. 0.3 to $0.4 \mathrm{eV}$ ). Lower current density and high barrier height of $\sim 0.7 \mathrm{eV}$ in the reference ITO electrode devices indicated the current injection is limited by this high electrode barrier [24, 25].

Fig. $3 \mathrm{~b}$ shows the charge carrier injection efficiency in the reference ITO and $\mathrm{COOH}-$ MWCNTs modified ITO OLEDs which was determined from the ratio of the measured current density in these devices to a space charge limited current density. It is noted that the 
injection efficiency in $\mathrm{COOH}-\mathrm{MWCNTs}$ modified ITO OLEDs is a few orders of magnitude higher at lower applied voltages. This would facilitate achieving OLED operation at very low voltages. For example a current density of $5 \mathrm{~mA} / \mathrm{cm}^{2}$ is injected at $\sim 3.7 \mathrm{~V}$ in the ITO/COOHMWCNT OLEDs in comparison to a reference ITO OLEDs which has a voltage which is $35 \%$ larger at $\sim 5 \mathrm{~V}$. It should also be noted that the band discontinuity of $0.4 \mathrm{eV}$ shown between the ITO/ COOH-MWCNT and TPD in Fig. 2(b) is further modified due to the high aspect ratio of the MWCNT. Much like in the case of electron field emission $[6,26]$. There will be field enhancement around the MWCNT which will further lower any barrier at the interface. Due to this very high injection, the effective mobility appears to attain a value of $10^{-}$ ${ }^{3} \mathrm{~cm}^{2} / \mathrm{Vs}$ or higher in the composite COOH-MWCNT modified ITO devices.

In addition to high charge carrier injection efficiency, there is a cusp observed from applied voltage of 4 to $7 \mathrm{~V}$ in the $\mathrm{J}-\mathrm{V}$ characteristics of COOH-MWCNT modified ITO OLED (Fig. 3c and 3d). The observation of this kind of cusp has been reported earlier in the literature as an anomalous current-voltage characteristic leading to a "negative differential resistance (NDR)" for different types of organic materials [27-29]. These were observed due the charge carrier transport through localised states formed in TPD (generally in organic semiconducting materials) [27]. The origin and the associated charge transport properties of these localised states are not well identified, but affected by the device thickness and temperature [27]. Furthermore, the higher current during this initial turning on of the device does not give rise to corresponding significant increase in light output and there is no correlation between the current in the NDR region and the electroluminescence characteristics. The reproducible nature of $\mathrm{J}-\mathrm{V}$ ruled out the possibility of the burnt-shunt effect reported in the devices in the literature [27] and this equally applies to the features observed with our COOH-MWCNT modified ITO OLEDs. Recently, devices with NDR have been reported with iodine or nitrogen doped TPD/Alq3 bilayer devices $[28,29]$. The 
NDR was not observed with iodine doped TPD single layer devices, but exhibited higher current density than that of undoped devices. This behaviour would be expected if the NDR is a minority carrier phenomenon where a high current density would mask such a phenomena occurring in a parallel pathway $[30,31]$. Higher current injection in single layer device was attributed to the charge transport through the hopping sites in TPD and the additional hopping sites created by the doping of iodine or nitrogen. In bilayer devices of TPD/Alq3, the appearance of NDR behaviour was explained by proposing the emission of phonons during the recombination process from the highly energetic holes travelling through doping induced hopping sites and electrons from Alq3 [28,29]. These phonons led to the scattering of charge carriers and decrease in the current with increasing applied voltage. In order to investigate the two step turn-on phenomena, attempts were made to fit Fowler-Nordheim model to the two turn on regions which resulted in poor fits, confirming that although the structure has a discontinuous buffer layer, the effect is composite, and the relevance of existing models is speculative and does not fully elucidate the phenomenon occurring in our films. [32]
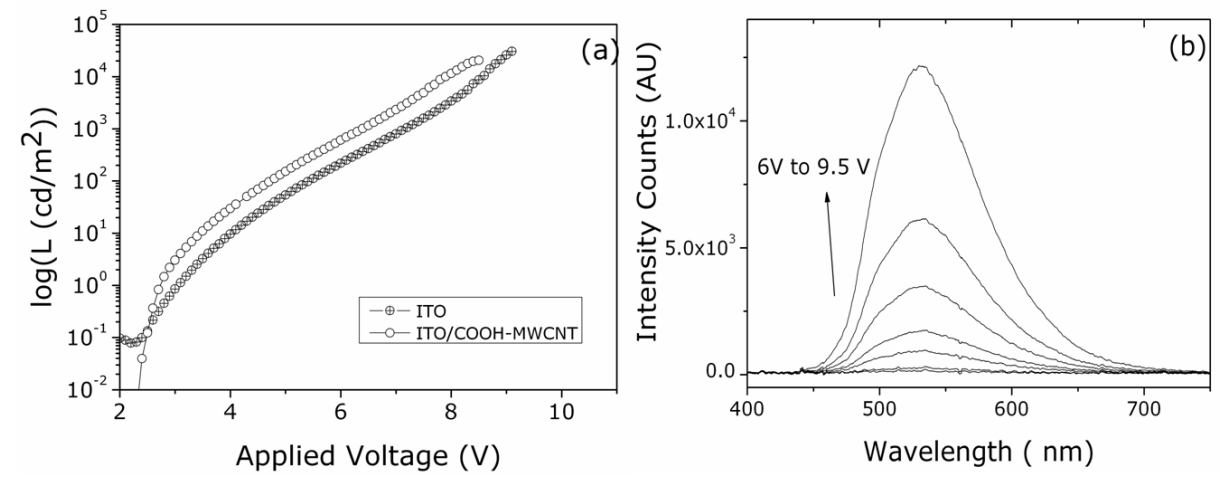

Figure 4. (a) Luminance vs voltage characteristics of reference ITO and ITO/COOHMWCNT electrode OLED and (b) Electroluminescence spectra of ITO/COOH-MWCNT OLEDs where the voltage is ramped from $6.0 \mathrm{~V}$ to $9.5 \mathrm{~V}$ (in steps of $0.5 \mathrm{~V}$ ). 
Fig. 4a shows the luminescence vs voltage characteristics of reference ITO and ITO/COOH-MWCNT OLED. Among these devices the reference device exhibits the highest luminance of $30,434 \mathrm{~cd} / \mathrm{m}^{2}(\sim 9.1 \mathrm{~V})$, while ITO/COOH-MWCNT OLED has a recorded highest luminance of $20,562 \mathrm{~cd} / \mathrm{m}^{2}(\sim 8.5 \mathrm{~V})$ respectively. It is also observed that the ITO/COOH-MWCNT OLED has a higher luminance than that of the reference ITO OLED at voltages between $3-8 \mathrm{~V}$. Since the device is operated under a continuous applied voltage mode with a step voltage of $0.1 \mathrm{~V}$, the device shows evidence of degradation due to joule heating with extended times. ITO/COOH-MWCNT OLED device turns-on $\left(1 \mathrm{~cd} / \mathrm{m}^{2}\right)$ around 2.8 $\mathrm{V}$ while the reference ITO OLED turns on at slightly higher voltage of 3.1. The light output of ITO/COOH-MWCNT OLED devices is not well correlated with the current densities through the region with lower voltage turn-on, similar to devices reported with NDR behaviour. For example, at the applied voltage of 5.3V, ITO/COOH-MWCNT OLED exhibits luminance of $235 \mathrm{~cd} / \mathrm{m}^{2}\left(71 \mathrm{~mA} / \mathrm{cm}^{2}\right)$, while the reference ITO OLED has its luminance of $\sim 84 \mathrm{~cd} / \mathrm{m}^{2}\left(2.45 \mathrm{~mA} / \mathrm{cm}^{2}\right)$; which are equivalent to $\sim 3$ times increase in light emission intensity and about $\sim 30$ times increase in current density. If we assume the external quantum efficiency of the device to be of the order of $1.5 \%$, then the expected increase in light emission intensity would be less than $50 \%$ (as opposed to $300 \%$ ). The huge increase in the light output will be discussed in terms of higher charge injection, more localised radiative recombination and better transport through the device structure as a result of the CNT incorporation as a hole injection layer.

Fig. $4 \mathrm{~b}$ shows the electroluminescence spectra of ITO/COOH-MWCNT electrode OLED which are characteristic of Alq3 OLED with the emission peak around $530 \mathrm{~nm}$. Since the electroluminescence from the ITO reference and ITO/COOH-MWCNT OLED are similar, the electroluminescence of reference ITO OLED was not shown in the figure for clarity (but available in the supporting information). Although the ITO/COOH-MWCNT OLED devices 
exhibit different exponent values in $\mathrm{J}-\mathrm{V}$ curves after turn on, the spectra at different applied voltages do not have a significant spectral shift or the appearance of new emission peaks compared with the references. These spectra confirm that the presence of COOH-MWCNT with the corresponding lower turn on region do not change the emission characteristics of Alq3 devices and the emission is confined to the Alq3 layer. This is also in agreement with earlier reports that the HTL TPD does not contribute to the light emission [16, 28].

(a)

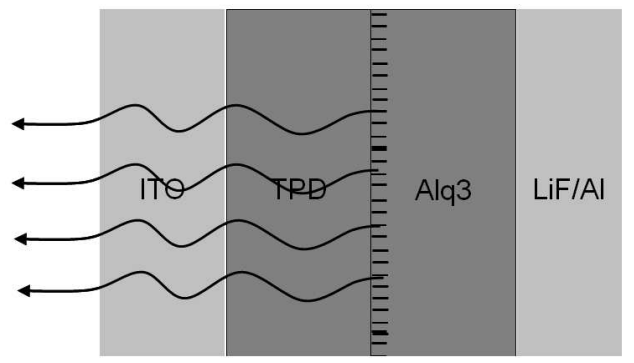

(b)

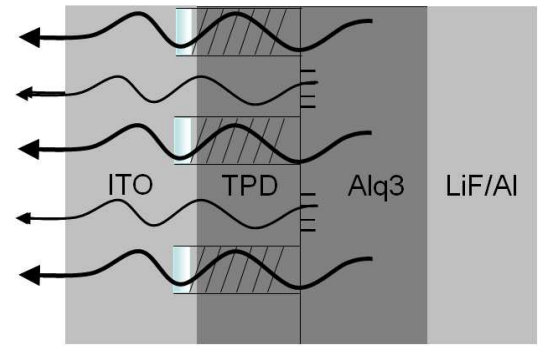

Figure 5. Schematic diagram of emission from (a) reference ITO electrodes and (b) ITO/COOH-MWCNT electrode OLEDs (Crossed lines refer to the area just above the COOH-MWCNT which may be responsible for the NDR-like behaviour; thick arrow represents the light output pathways from the NDR-like regions).

A possible model to explain this increase in current and light output at a given voltage is shown schematically in Fig. 5. In the reference ITO device, electrons pile up at the Alq3/TPD interface due to the large barrier height between the TPD and the Alq3 (see Fig 2a). These electrons near the interface recombine with holes injected from the TPD and are replenished by electrons injected from the cathode. The negative space charge at the interface reduces the electric field in the Alq3 and the electron current since few electrons get over the barrier and enter the TPD. In OLED with ITO/COOH-MWCNT electrodes (Fig 2b), due to the relatively small hole barrier of $\sim 0.4 \mathrm{eV}$ compared to that of ITO reference electrodes, more and more holes are injected towards the TPD/Alq3 layers. The hole space charge lowers 
the electron barrier. This barrier height reduction enables more electrons to enter the TPD layer and recombine with the holes. This leads to higher electron current through the Alq3 and higher total current. This assumption is supported by the observation of lower external quantum efficiency, because there is now more non-radiative recombination taking place in the TPD layer than that of reference ITO electrode OLED. This can be balanced in the future by increasing the electron transport in the device or moving the recombination pathway away from the TPD to the Alq3. High electron concentration throughout the Alq3 layer results in recombination more evenly spread in the Alq3 layer. This leads to the observation of higher overall luminance in ITO/COOH-MWCNT electrode OLED at any given applied voltage compared with the reference ITO electrode devices. Earlier reports from our group on hole only devices of ITO/SWCNT/TPD(100 nm)/Al suggests that the TPD morphology is modified by the presence of COOH-MWCNT on the top of ITO [9]. TPD on to the top of ITO appears to have a more crystalline regime just above the COOH-MWCNT, compared to that of TPD on the top of the ITO. Fig 5b depicts the situation where the TPD morphology is modified due to the presence of COOH-MWCNT. In the TPD crystalline phases, the lower barrier for electrons may enable electrons to enter the TPD where they may recombine with holes non-radiatively. Therefore, the build-up of electrons at the interface is prevented (Fig 5) and there is a subsequent increase in electric field within the Alq3.

The reference ITO and ITO/COOH-MWCNT OLED exhibit a high current efficiency of $\sim 18 \mathrm{~cd} / \mathrm{A}$ at $\sim 8 \mathrm{~V}$. The lower efficiencies at lower voltages could be explained by the fact that in the ITO/COOH-MWCNT OLEDs an increase in its light output ( 3 times) is not proportional to the increase in the injected current density ( $\sim 30$ times). This disproportionate increase in ratio of light output over injected current density leads to the lower EQE (Fig. 6) below $200 \mathrm{~mA} / \mathrm{cm}^{2}$. As the EQE is proportional to the ratio of the light output to the total injected current, a voltage independent EQE of device reference ITO may show that the 
conduction process is not injection-limited [19]. While the barrier height of $\sim 0.7 \mathrm{eV}$ at the ITO/TPD suggests the current is injection limited in the reference devices [24], the voltage independent EQE could also be due to a balanced of injection in holes and electrons. While the voltage dependent EQE of the ITO/COOH-MWCNT OLED may initially appear to indicate that the conduction process is injection limited (electron in this case), the barrier height of $\sim 0.4 \mathrm{eV}$ at the ITO/ COOH-MWCNT interface and SCLC fitting suggests that the conduction process is not. This voltage dependent EQE is likely to be influenced by the enhanced hole injection with respect to applied electric field in the ITO/COOH-MWCNT OLEDs. The basic conclusion of our model, enhanced hole injection efficiency with ITO/COOH-MWCNT is corroborated by the EQE measurements.

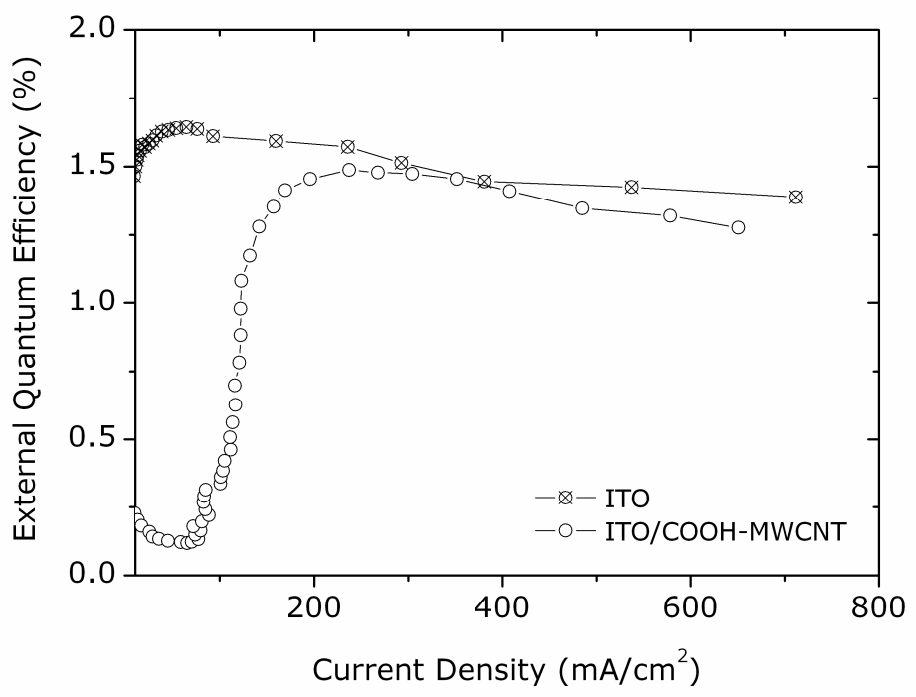

Figure 6. External quantum efficiency of reference ITO and ITO/COOH-MWCNT electrode OLED. Lower efficiency in the NDR like region is attributed to the disproportionate ratio of current injection (30 times) to luminance (3 times) in ITO/COOH-MWCNT OLED

The lower EQEs at lower current densities are attributed to the non-optimum carrier recombination in the Alq3 layer and due to the lower level of electron mobility and lower electron injection compared to that of the improved holes in the ITO/COOH-MWCNT 
OLED. There is no one to one correlation to the increase in light output to the increase in holes passing through the material. We believe this is due to the unavailability of sufficient electrons [20]. It may be possible that some electrons might get in to the TPD regions due to SCLC effects as discussed earlier and might recombine non-radiativley. It is more important that the radiative recombination only occurs in Alq3 and not in TPD.

\section{Conclusions}

The presence of COOH-MWCNT at the anode increases hole injection, which has an immediate impact even at low voltages and can be used to mitigate against previous device architectures which were limited by poor hole injection from ITO reference devices. Though the presence of COOH-MWCNT creates localised states and space charge in the TPD that has the effect of lowering the barrier to electron flow between the Alq3 and TPD, the radiative recombination takes place within the Alq3 and the emission spectrum is not affected. The appearance of NDR-like phenomena and its effective utilisation in device performance needs further detailed investigation and can be a useful tool for future designers.

\section{Acknowledgements}

This research was partly funded by a portfolio partnership award by the EPSRC, U.K., and by E.ON AG, as part of the E.ON International Research Initiative. Responsibility for the content of this publication lies with the authors.

\section{References}

[1] Baldo MA, O’Brian DF, You Y, Shoustikov A, Silby S, Thomson ME, Forrest SR.

Highly efficient phosphorescent emission from organic electroluminescent devices. Nature 1998; 395: 151-4. 
[2] Weaver MS, Bradley DDC. Organic electroluminescence devices fabricated with chemical vapour deposited polyazomethine films. Synth. Met. 1996; 83b: 61-6.

[3] Gao XC, Cao H, Huang CH, Li BG, Ibrahim K, Liu FQ, Umitani S.

Electroluminescence from both a light-emitting layer and hole transport layer: spectral evidence for charge carrier tunneling injection. Chem. Phys. Lett. 1998; 297: 530-6.

[4] Chen BJ, Lai WY, Gao ZQ, Lee CS, Lee ST, Gambling WA. Electron drift mobility and electroluminescent efficiency of tris(8-hydroxyquinolinolato) aluminium. Appl. Phys. Lett. 1999; 75: 4010-12.

[5] Dai L. Light-emitting polymers and carbon nanotube electron emitters for optoelectronic displays. Smart Mater. Struct. 2002; 11: 645-51.

[6] Hatton RA, Blanchard NP, Stolojan V, Miller AJ, Silva SRP. Nanostructured copper phthalocyanine-sensitized multiwall carbon nanotube films. Langmuir 2007; 23: 6424-30. [7] Blanchard NP, Hatton RA, Silva SRP. Tuning the work function of surface oxidised multi-wall carbon nanotubes via cation exchange. Chem. Phys. Lett. 2007; 434 : 92-5. [8] Aguirre CM, Auvray S, Pigeon S, Izquierdo R, Desjardins P, Martel R. Carbon nanotube sheets as electrodes in organic light-emitting diodes. Appl. Phys. Lett. 2006; 88 183104.

[9] Wang GF, Tao XM, Wang RX. Fabrication and characterization of OLEDs using PEDOT:PSS and MWCNT nanocomposites. Comp. Sci and Tech 2008; 68: 2837-41. [10] Tan LW, Hatton RA, Latini G, Shannon JM, Silva SRP. Modification of charge transport in triphenyldiamine films induced by acid oxidized single-walled carbon nanotube interlayers. Nanotechnology 2008; 19: 485706.

[11] Liu D, Fina M, Guo J, Chen X, Liu G, Johnson SG, Mao SS. Organic light-emitting diodes with carbon nanotube cathode-organic interface layer. Appl. Phys. Lett. 2009; 94: 013110. 
[12] Davids PS, Campbell IH, Smith DL. Device model for single carrier organic diodes. J. Appl. Phys. 1997; 82:6319-26.

[13] So SK, Tse SC, Tong KL. Charge Transport and Injection to Phenylamine-Based Hole Transporters for OLEDs Application. J. Disp. Tech. 2007; 3: 225-232.

[14] Rose A. Space-Charge-Limited Currents in Solids. Phys. Rev. 1955; 97: 1538-44.

[15] Lambert MA. A simplified theory of two-carrier, space-cgarge-limited current flow in solids. .RCA Review 1959; 20: 682.

[16] Burrows PE, Shen Z, Bulovic V, McCarthy DM, SR Forrest, Cronin JA, Thompson ME. Relationship between electroluminescence and current transport in organic heterojunction light-emitting devices. J. Appl. Phys. 1996; 79: 7991-8006.

[17] Antoniadis H, Miller JN, Roitman DB, Cambell IH. Effects of hole carrier injection and transport in organic light-emitting diodes. IEEE Transactions on Electron Devices 1997; 44: 1289-94.

[18] Ioannidos A, Facci JS, Abkowitz MA. Evolution in the charge injection efficiency of evaporated Au contacts on a molecularly doped polymer. J. Appl. Phys. 1998, 84: 143945.

[19] Tse SC, Tsang SW, So SK. Polymeric conducting anode for small organic transporting molecules in dark injection experiments. J. Appl. Phys. 2009; 100: 063708,

[20] Shen Y, Jacobs DB, Mallaras G, Koley G, Spencer MG, Ioannidis. Modification of Indium Tin Oxide for Improved Hole Injection in Organic Light Emitting Diodes. Adv. Mater. 2001; 13: 1234-1238.

[21] Kaliniwski J, Palilis LC, Kim WH, Kafafi ZH. Determination of the width of the carrier recombination zone in organic light-emitting diodes. J. Appl. Phys. 2003; 94: 7764-8. 
[22] Lambert MA, Mark P. Current Injection in Solids. Academic, New York, 1970.

[23] Wu CC, Chun JKM, Burrows PE, Sturm JC, Thomson ME, Forrest SR, Register AR. $\operatorname{Poly}(p$-phenylene vinylene)/tris(8-hydroxy) quinoline aluminum heterostructure light emitting diode. Appl. Phys. Lett.1995; 66: 653-5

[24] Hatton RA, Blanchard NP, Tan LW, Latini G, Cacialli F, Silva SRP. Oxidised carbon nanotubes as solution processable, high work function hole-extraction layers for organic solar cells. Org. Electron. 2009; 10: 388-95.

[25] Campbell AJ, Bradley DDC, Laubender J, Sokolowski M. Thermally activated injection limited conduction in single layer N,N8-diphenyl-N,N8-bis.3-methylphenyl.1-18biphenyl-4,48-diamine light emitting diodes. J. Appl. Phys.1999; 86: 5004-11.

[26] Miller AJ, Hatton RA, Silva SRP. Interpenetrating multiwall carbon nanotube electrodes for organic solar cells. Appl. Phys. Lett. 2006; 89:133117.

[27] Berlab S, Brutting W, Schwoerer M. Anomalous current-voltage characteristics in organic light-emitting devices. Synth. Met. 1999; 102:1034-37.

[28] Fanga YK, Chiang YT, Chen SF, Lin CY, Hou SC, Hung CS, Tsai TY, Chang SH, Chou TH. Observation of room temperature negative differential resistance (NDR) in organic light-emitting diode with inorganic dopant. J. Phys. \& Chem. Solids 2008; 69: $738-41$.

[29] Chou TH, Chen SF, Fang YK, Hou CS, Lin FS, Lin CY. Significantly Improved Luminance of Organic Light-Emitting Diodes by Doping Iodine and Nitrogen Treatment. Jpn. J. Appl. Phys. 46; 2007 :2753-57. 
[30] Bhattacharyya S, Henley SJ, Mendoza E, Gomez-Rojas L, Allam J, Silva SRP.

Resonant tunnelling and fast switching in amorphous-carbon quantum-well structures.

Nat. Mater. 2006; 5: 19-22.

[31] Chan KK, Silva SRP, Amartunga G. Electronic properties of semiconducting diamond-like carbon-diamond . Thin Solid Films 1992; 212: 232-239.

[32] Oey CC, Djurixic AB, Kwong CY, Cheung CH, Chan WK, Nunzi JM, Chui PC. Nanocomposite hole injection layer for organic device applications. Thin Solid Films $2005 ; 492: 253-258$. 